\title{
Characterizing enterotypes in human metagenomics: a viral perspective
}

$2 \quad$ Li Song ${ }^{1}$, Lu Zhang ${ }^{2 *}$, Xiaodong Fang ${ }^{1^{*}}$

$3{ }^{1}$ College of Life Sciences, University of Chinese Academy of Sciences, Beijing 100049, China

$4 \quad{ }^{2}$ Department of Computer Science, Hong Kong Baptist University, Hong Kong

*Correspondence:

6 Corresponding Authors

7 fangxd@bgi.com, ericluzhang@hkbu.edu.hk

8 Keywords: Gut metagenomics, Virome, Enterotype, Biomarker, Perspective

\section{Abstract}

The diversity and high genomic mutation rates of viral species hinder our understanding of viruses and their contributions to human health. Here we investigated the human fecal virome using previously published sequencing data of 2,690 metagenomes from seven countries. We found that the virome was dominated by double-stranded DNA viruses, and young children and adults showed dramatic differences in their fecal enterovirus composition. Beta diversity showed there were significantly higher distances to centroids in individuals with severe phenotypes, such as cirrhosis. In contrast, there were no significant differences in lengths to centroids or viral components between patients with mild phenotypes, such as hypertension. Enterotypes showed the same specific viruses and enrichment direction after independent determination of enterotypes in various projects. Confounding factors, such as different sequencing platforms and library construction, did not result in a batch effect to confuse enterotype assignment. The gut virome composition pattern could be described by two viral enterotypes, which supported a discrete, rather than a gradient, distribution. Compared with enterotype 2, enterotype 1 had a higher viral count and Shannon index, but a lower beta diversity, indicating more resistance to the external environment's harmful effects. Disease was usually accompanied by a viral enterotype disorder. However, a sample outside of the enterotyping mathematical space of enterotype database did not necessarily indicate sickness. Therefore, the background context must be carefully considered when using a viral enterotype as a biomarker for disease prediction. The disease, second only to the enterotype, explains significant variation in viral community composition, implying that double-stranded DNA is relevant to human health. Our results of investigating a baseline viral database highlight important insights into the virome composition of human ecosystems, and provide an alternate biomarker for early disease screening.

\section{$31 \quad 1 \quad$ Background}

In recent years, many studies have shown that viral colonization in the human body is highly related to human health and life. Cross-species virus transmission poses an extraordinary threat to human and animal health (Daszak et al., 2000). With advanced sequencing technology, the primary material for viral research has become viral genomes (virome), which enable viral identification and classification at the molecular level (Fujimoto et al., 2020; Gregory et al., 2020). The success of virome studies greatly relies on high-quality viral genomes (Minot et al., 2011). However, viruses are 
sequencing are labor-intensive and inefficient (Reddy et al., 2015), thus severely preventing the comprehensive and intensive study of viruses.

The strategy of assembling the viral genome involves a comprehensive and in-depth analysis of the virome. David and colleagues launched the "Uncovering Earth's virome" project to build The Integrated Microbial Genome/Virus (IMG/VR) database in 2016(Paez-Espino et al., 2016, 2017, 2019; Roux et al., 2021). Recently, data of 28,060 metagenomes were used to mine 142,809 human gut viruses, and Gubaphage was found to be the second common virus branch in the human gut(Camarillo-Guerrero et al., 2021). These projects opened the prelude to the construction of viral genome database and laid the foundation for a comprehensive analysis of the human gut virome(Gregory et al., 2020). Disease is associated with the gut virome, but studies have ignored the importance of viral sequencing information in massive metagenome sequencing data. The construction of the viral genome database has enabled detailed research on the human gut virome.

An enterotype is a cluster of microbes in the human gut and it describes the distributional of the human gut microbial community(Arumugam et al., 2011). Multiple studies have reported that there are two dominant enterotypes, which correspond to the individuals' preference for digesting plant fiber or animal meat (Costea et al., 2017). The gut is an ecosystem, and the enterotype summarizes its microbial characteristics using mathematical methods(Arumugam et al., 2011; Holmes et al., 2012), but such knowledge is insufficient (Jeffery et al., 2012). Research on the composition patterns and function of the gut microbiome will significantly improve our understanding of its relationship with health and disease(Knights et al., 2014). Enterotypes can be used for gut microbial analysis, to inform disease treatment and prevention strategies, and may also provide a theoretical basis for diet therapy. The relationship between viral enterotypes and the human disease status is still largely unknown. Whether enterotypes can be used as biomarkers for predicting the disease status requires further research.

In this study, we collected previously published human metagenomic sequencing data, conducted sample quality control through a fast pipeline, identified virus species, and determined viral abundance. Furthermore, we established a baseline database of the human gut virome based on 2,690 metagenomes. We demonstrated the relationship between virus species and abundance in various ethnicities, countries, and diseases using different DNA library construction methods and sequencing platforms, and analyzed the association between viral community diversity and disease. Viral enterotypes were assigned by the Dirichlet multinomial mixture model (DMM). We independently identified enterotype-specific viral operational taxonomic units (vOTUs) for each dataset and resolved the inter-relationships among enterotypes from different projects by comparing the abundance of enterotype-specific viruses. Further, we compared the ecological diversity of viruses between different enterotypes, and evaluated the correlation of viral enterotype disorders and their diversity with diseases. The results of study elucidate the relationship between enteroviruses and human health in a large population and highlight the decisive role of viruses as molecular markers in identifying high-risk individuals. Viral research is likely to make an indispensable contribution to improving human health.

\section{$78 \quad 2 \quad$ Materials and methods}

\subsection{Choosing an alignment method}

80 Alignment and assembly methods are used to detect viruses and estimate their abundance.

81 MetaPhlAn(Segata et al., 2012)and its upgraded version, MetaPhlAn2(Truong et al., 2015), are

82 alignment tools that use marker genes for alignment and have achieved great success in bacterial 
83

84

85

86

87

88

89

90

91

92

93

94

95

96

97

98

99

100

101

102

103

104

105

106

107

108

109

110

111

112

113

114

115

116

117

118

119

120

121

122

123

124

125

126

genome alignment. However, many viral genomes do not have marker genes, and therefore, this strategy is not useful for viral classification and abundance estimation. Virome(Eric Wommack et al., 2012), VirSorter(Roux et al., 2015), and VirFinder(Ren et al., 2017) use assembly methods to classify viruses and calculate abundance, but these tools require a large amount of computing resources and time and therefore cannot be applied to large projects. Some recently developed alignment tools, such as ViromeScan(Rampelli et al., 2016), VIP(Li et al., 2016), and HoloVir(Laffy et al., 2016) have been shown to perform well for bacterial genomes. However, they are impractical for aligning viral reads to genomes. Moreover, many software tools are for online use, which means that they are unsuitable for large-scale projects. VirMap(Ajami et al., 2018) software developed for processing protein and genome data can provide good results. It can be accurately identify the virus species regardless of the sequencing depth. However, this software involves substantial computing resources. After comparing the advantages and disadvantages of different software(Ajami et al., 2018), we finally chose FastViromeExplorer(Tithi et al., 2018), a software based on k-mer alignment used by Kallisto(Bray et al., 2016). This software maps all reads to the reference and then uses the expectation-maximization algorithm to estimate the virus species and their corresponding abundance.

\subsection{Data collection and processing}

We downloaded all data from the National Center for Biotechnology Information (NCBI) sequence read archive (SRA). The SRA numbers for each project are shown in Supplementary Table 1. We only chose pair-end data from projects sequenced by the Illumina HiSeq 2000 or 2500 platforms. After processing the original data sample (Supplementary Figure 1) using Trimmomatics(Bolger et al., 2014)to remove the raw data and adapters of low-quality reads, we detected and removed contamination from the host's DNA and RNA data, and discarded the unpaired reads. Finally, we used FastViromeExplorer software to align reads to IMG/VR v2.

\subsection{Viral contig taxonomic annotation}

We used Glimmer3 toolkit Version 3.02b(Delcher et al., 2007) to predict and extract the open reading frame of viral contigs with a minimum length threshold of 100 amino acids. The protein sequences were aligned to the UniProt TrEMBL database as of February 2021(Bateman et al., 2021) using BLASTX(Boratyn et al., 2012). The major voting system was then used as described previously to ascertain the family of a viral contig. A contig needed to be supported by five proteins to be considered as successful assignment; otherwise, the assignment was considered a failure. When a virus sequence was annotated to multiple families in taxonomic assignment, we choose the family with the largest proteins. When multiple families have the same number of proteins, the size of the accumulated E-value (BLASTX alignment) of all proteins was compared.

\subsection{Calculation of ecological diversity}

We first used Tximport(Soneson et al., 2015) R package to read the original abundance information of the virus (the output of Kallisto) from each project. The "betadiver" in the Vegan R package was used for calculating alpha and beta diversity. For alpha diversity, we first transformed the abundance information into integers and then used the "rrarefy" function to normalize abundance. We then used the "estimate," "diversity," and "specnumber" functions to obtain various measurement values of alpha diversity. We used the "RLE" method embedded in "calcNormFactors" to normalize raw abundance for beta diversity. We used the "Hellinger" method in "Decostand" to transform the data and eliminate false similarities caused by many viruses whose abundance was 0 . When the abundance of many viruses in the two samples were 0 , some algorithms might consider them to have similar abundance distribution and conclude that they were close to each other. The real reason may 
127 be that many viruses have not been detected. We used the "betadiver" and "betadisper" functions to

128 obtain beta diversity, and then used Adonis2 to analyze the viral ecological differences between cases and controls, and corrected them with raw data size. The Kruskal test was used to determine whether

130 there was a significant difference in the distance from the centroid between cases and controls.

131 Tukey's honestly significant difference test was used to determine differences in variance within and

132 between groups.

\section{$133 \quad 2.5 \quad$ Enterotyping and MaAsLin2 analysis}

134 We used the DMM method to determine viral enterotypes in each project independently. Enterotypes

135 were assigned using the "DirichletMultinomial" $\mathrm{R}$ package, with predetermined parameters of 1 to 10

136

137 enterotypes, and enterotype data from each project were run 10 times. The smallest Laplace value corresponding to the number of enterotypes was considered as the optimal result. MaAsLin2 (huttenhower.sph.harvard.edu/maaslin2) analysis was used to determine the specific vOTUs associated with enterotypes, with correlations considered significant at the 5\% level (after multiple testing correction). We also applied the envfit function in Vegan to estimate the effect size of the structural variance explained by factors such as enterotype and disease.

\section{$142 \quad 3 \quad$ Results}

\section{$143 \quad 3.1$ Sequencing data and summarization}

144 We collected $12.36 \mathrm{~TB}$ of metagenomic sequencing data from 18 previously published projects 145 (Supplementary Tables 1 and 2). We selected data from 2,690 metagenome samples of high quality 146 for the subsequent analysis (Supplementary Figure 1 and Supplementary Table 1), of which 1,092 147 were samples were from women, 859 were from men, and 739 were from unknown sex. The length of sequencing reads from each sample were 2.26 to $8.55 \mathrm{G}$ (Supplementary Table 1), and approximately $10 \%$ of strictly filtered reads were aligned against IMG/VR v2 viral sequences (Supplementary Table 3). We obtained 2,690 metagenome samples by choosing paired-end sequencing data from the Illumina HiSeq 2000 and 2500 platforms and excluding projects with a small data size $(<1 \mathrm{G})$.

154 We annotated the geographic locations of the included projects on the basis of their predominant 155 samples (Figure 1A). Because there were no specific sampling coordinates, each project was located by country. We annotated the viral taxonomy at the family level based on the protein sequence similarities (Minot et al., 2013; Hannigan et al., 2015). Approximately 50\% of the viral genomes failed taxonomic assignment (Figure 1B), and double-stranded (ds) DNA viruses, such as Siphoviridae, Myoviridae, and Podoviridae, were the dominant enteroviruses as previously reported (Zuo et al., 2020). The density peak was close to zero, which indicated that the viruses were rarely shared among individuals (Supplementary Figure 2). The samples from Finland were outliers in the PCoA and tSNE plots (Figure 1C and D) because of the low viral diversity (Supplementary Figure 3). This finding might be explained by age. The average age of individuals in the Finland project was 1.5, and their gut communities did not reach stable states. Although the samples from the other six countries showed substantial variability in the PCoA and tSNE plots (Figure 1C and D), they belonged to the same cluster, especially the samples from the studies conducted in China. The studies from China had the most individuals, and the samples were spread over almost the entire plot. In the tSNE plot, we found that the samples from the USA and Peru were clustered in a local region, which indicated that the gut virome showed characteristics of geographical distribution. 
$170 \quad$ [insert figure 2 here]

171 To study the distribution characteristics of the viral species in samples with different phenotypes, we

172 divided all samples from studies with a case-control design into three categories. These categories of

173 controls, cases, and all represented healthy people, patients with various diseases, and all individuals,

174 respectively. As more samples were included, the number of viral species showed exponential

175 growth, with no significant difference between cases and controls until samples from 100

176 individuals were included (Figure 2A). After including 100 individuals, the "case" curve showed a

177 steep increased viral count. As expected, a significant increment in the number of viral species was

178 observed when the number of samples was increased in the "all" curve. However, the three growth

179 curves were essentially parallel (Figure 2A), which suggested that the overall number of viruses in

180 the patient population after viral community disruption was limited. More interestingly, the "case"

181 and "all" curves overlapped with each other after $\sim 1000$ samples. The reason for this finding could be

182 that the case population contained all species of viruses in the control population. When we

183 compared the growth curves of different projects, we found that the curves for Finland, Peru, and

184 Chinese populations with cirrhosis had significant differences (Figure 2B). The samples from the

185 Finland project were obtained from only 1.5-year-old children, at which age the enterovirus

186 community is not well established. It is unclear why the number of viral species in Peru samples were

187 small at the beginning of the curve. The dramatic increase in the number of viruses in the Chinese

188 population with cirrhosis may be due to severe disruption of the enterovirus community. We used

189 unique species in cases and controls to define group-specific viruses and compared the change in the

190 proportion of unique viral species between cases and controls (Supplementary Table 4). We found

191 that the mean proportion of viruses in case samples was $26 \%$ and in control samples was $14 \%$.

192 Among all samples, the proportion of viruses that were unique to cases was $23 \%$. Each case

193 individual had an average of 10.99 viruses, and the ratio of viruses that were unique to controls was

$1944 \%$, and each control individual had an average of 2.43 viruses. Overall, there was an enrichment of

195 viruses in cases.

Table 1. Beta diversity for measuring the sample distance in projects with a case-control design.

\begin{tabular}{lcccc}
\hline Project & $\begin{array}{c}\text { Adonis2 for } \\
\text { disease }\end{array}$ & $\begin{array}{c}\text { Adonis2 for } \\
\text { raw data }\end{array}$ & ANOVA & $\begin{array}{c}\text { Kruskal } \\
\text { test }\end{array}$ \\
\hline Sweden T2D* & $5.00 \mathrm{E}-03$ & $1.00 \mathrm{E}-03$ & $6.34 \mathrm{E}-03$ & $1.62 \mathrm{E}-03$ \\
China cirrhosis & $1.00 \mathrm{E}-03$ & $1.00 \mathrm{E}-03$ & $3.91 \mathrm{E}-09$ & $8.41 \mathrm{E}-14$ \\
China rheumatoid arthritis & $2.40 \mathrm{E}-02$ & $1.50 \mathrm{E}-02$ & 0.86 & 0.48 \\
Austria carcinoma & $1.00 \mathrm{E}-03$ & 0.55 & $5.29 \mathrm{E}-04$ & $7.89 \mathrm{E}-05$ \\
China colorectal cancer & $4.00 \mathrm{E}-03$ & $3.10 \mathrm{E}-02$ & 0.12 & $9.71 \mathrm{E}-03$ \\
China hypertension & 0.08 & 0.25 & 0.13 & 0.08
\end{tabular}




\begin{tabular}{lcccc} 
China coronary heart disease & $1.00 \mathrm{E}-03$ & 0.81 & $4.15 \mathrm{E}-02$ & $1.79 \mathrm{E}-02$ \\
China T2D discovery & $2.60 \mathrm{E}-02$ & $3.50 \mathrm{E}-02$ & $1.75 \mathrm{E}-02$ & 0.15 \\
China T2D validation & $1.00 \mathrm{E}-03$ & $2.90 \mathrm{E}-02$ & 0.38 & 0.45 \\
China obesity & 0.06 & 0.37 & 0.78 & 0.78 \\
\hline *Type 2 diabetes (T2D). & & & &
\end{tabular}

\section{$197 \quad 3.2 \quad$ Relationship of ecological diversity of viruses and disease}

198 The beta diversity of a microbial community is usually used to evaluate dynamic changes in an 199 ecosystem (Koleff et al., 2003). A comparison of the results of projects with a case-control design revealed that the degree of imbalance in the viral community composition was related to the severity of the disease phenotype. An example of this finding is that the viral community in patients with cirrhosis (Figure 3A) was significantly different from that in healthy people (Adonis2, $p=0.001$, adjusted for raw data size). Comparison of the distance to the centroid between patients and healthy individuals by the Mann-Whitney $U$ test showed a significant dissimilarity (Figure 3B). Specifically, patients had a significantly larger distance than healthy individuals, which indicated that patients had a considerably disordered viral community. In contrast, we did not detect a significant difference between patients and healthy individuals in the hypertension project (Adonis2, $\mathrm{p}=0.08$, Figure 3C). We also compared the distance to the centroid for each pair of three cohorts (Figure 3D), and a significant difference was found only between patients with hypertension and healthy individuals (Wilcoxon, $\mathrm{p}=0.036$ ).

We further investigated statistical differences in gut viral composition between case and control samples from various aspects to investigate changes in the viral community across different phenotypes. Using Adonis2, we found a significant difference in enteroviruses between cases and controls expect hypertension and obesity (Table 1), which suggested that their gut viral community was less affected by the disease state. Consistently, in cases with relatively mild phenotypes, such as hypertension or obesity, there was no noticeable differences in body metabolism compared with the controls. An analysis of variance (ANOVA) was used to determine whether there was a significant difference between two centroids (to test the component of viruses) between cases and controls. We found that the cirrhosis and cancer cohorts showed a substantial difference between two centroids (Table 1). The Kruskal-Wallis test was performed to determine whether the distance to the centroid in principal coordinates analysis was significantly different between the case and control groups, and the results were consistent with those of ANOVA. Compared with the controls, cases with more severe phenotypes, such as cirrhosis and cancer, showed substantial differences in gut viral composition (Table 1), whereas cases with relatively mild phenotypes, such as hypertension, showed no significant differences.

226 [insert figure 3 here]

\section{$227 \quad 3.3 \quad$ Characterizing viral enterotypes}

228 The characteristics of enterotypes of the gut virome were the focus of this study. Data on enterotypes are generally used to help adjust population stratification in Metagenome-wide association studies 
(MWAS) analysis (Wang et al., 2012). The correlation between enterotypes and disease phenotypes has received much attention in this field. The DMM method is commonly used for determining enterotypes of the gut microbiome and is more effective than the partitioning around medoids (Ding and Schloss, 2014). Different library construction methods, sequencing platforms, and other factors may lead to false-positive assignment of enterotypes. To avoid this situation, we adopted a projectindependent strategy for determining enterotypes. There were two or three enterotypes in most projects, while some projects only had one enterotype (Figure 1A, Table 2, Supplementary Figure 4). Enterotypes with the same intrinsic composition pattern were considered as the same. We used Maaslin2 to discover enterotype-specific vOTUs and then determined their enrichment direction on the basis of mean abundance. Similar enterotypes had the same specific vOTUs and the same enrichment trend. We manually classified enterotypes in all of the projects into three groups (Table 2 , Supplementary Table 5). Enterotypes 1 and 2, which are the two major types, were widely distributed in all projects, which indicated that these two types of enterotypes were common across the project populations. However, enterotype 3 was rare. Unclassified individuals were not able to be confidently assigned to enterotype 1 or 2 .

A permutation test was performed to demonstrate the validity of manual classification, which involved randomly paired enterotypes from different projects. We assumed that paired enterotypes had the same specific vOTUs and enrichment directions. We assigned a lower error rate to paired enterotypes if they had more identical vOTUs and similar enrichment trends. We repeated pairing 5 million times to obtain the distribution of pairing scores. These scores showed that our manually classified enterotypes had the lowest error rate (Figure 4A). Moreover, random pairing supported the three major enterotypes. Enterotypes 1- and 2-specific vOTUs were dominant (Figure 4B). The same enterotype-specific vOTUs with highly consistent enrichment trends indicated that the enterotypes from different projects had a similar pattern of virome composition (Figure 4B). Different DNA processing methods, sequencing platforms, ethics, age, and other confounding factors did not affect the identification of viral enterotypes. The vOTUs that were specific to unclassified enterotypes appeared complex. They intersected with either enterotype 1 or 2. Enterotype 3-specific vOTUs in different projects were less concordant than enterotypes 1- and 2-specific vOTUs.

258 [insert figure 4 here]

The microbiome is an ecosystem, the stability of which is reflected by the diversity of species in the system. As a species becomes more prosperous and uniform, the system's diversity increases and it becomes more resistant to the effects of the external environment(Keesing et al., 2010). There are two dominant enterotypes in the viral community (Zuo et al., 2020), one of which has a high alpha diversity. The results of our study are remarkably close to expected results. Although the viral count varied among samples from different projects, enterotype 1 across the samples had more viruses than enterotype 2 (Figure 4C). A higher value of the Shannon index and a smaller sample distance in enterotype 1 , compared with enterotype 2 , indicated its more stable composition pattern. We found that more individuals were categorized as enterotype 1 than enterotype 2 (1204 vs. 716). By comparing the proportion of healthy samples with the two enterotypes, we found that individuals who were categorized as enterotype 2 had a higher risk of being sick than those who were categorized as enterotype 1 (odds ratio: 1.38 , Fisher's exact test, $\mathrm{p}=0.01$ ). We observed an interesting finding when we compared samples from the cirrhosis project and the Sweden motherchild project. The third enterotype had the most discrete sample distribution in the cirrhosis project, and a higher viral count and Shannon index compared with the Sweden mother-child project (Figure 3C). In contrast, the third enterotype had a large sample distance and the lowest viral count and Shannon Index in the Sweden mother-child project. The cases in these two projects had 
276 diverse medical conditions. Specifically, the case cohort in the cirrhosis project had disordered gut

277 virome due to the disease, which explains why the number of viruses in the samples did not decrease.

278 In contrast, children in the Swedish mother-child project lacked a stable gut virome and had a lower

279 viral count, which suggested that enterotype 3 in the samples of this project was not caused by any

280 disease.

281 The viral enterotype may play a dominant role in influencing the structural variance of the gut virome

282 via a variety of factors. The Adonis test was used to determine the significance of viral enterotypes.

283 The results were significant in all projects. Our results explain most of the structural variance in the

284 gut virome (Figure 4D). In the Peru and cirrhosis projects, the Adonis R squared values were 0.62

285 and 0.57, respectively. Age, disease, BMI, raw data, and sex were not significant factors affecting

286 viral enterotypes in most projects, but Adonis $\mathrm{p}$ values reached significance in several projects.

287 Disease was the second most significant factor in the projects, which suggested that illness had a

288 higher ability to reshape the gut microbiome than other factors. Characterizing the interaction

289 between the gut virome and external stimuli was complex. Whether a single factor has a particular

290 contribution requires consideration of the context of this factor. An example of this situation is that,

291 in liver cirrhosis, the association between the gut virome and age was strong, but it was not

292 significant for diabetes.

293 Table 2: Manually categorized results for each project.

\begin{tabular}{llll}
\hline Enterotype & Enterotype 1 & Enterotype 2 & Enterotype 3 \\
\hline Denmark no phenotype & GP1 & GP2 & - \\
China cirrhotic & GP2 & GP1 & GP3 \\
Sweden mother-offspring pair & GP3 & GP2 & GP1 \\
China rheumatoid arthritis & GP1 & GP2 & - \\
Austria carcinoma & GP1 & - & GP2 \\
UK no phenotype & GP1 & GP2 & - \\
China colorectal cancer & GP1 & GP2 & - \\
China hypertension & GP2 & GP3 & - \\
China coronary heart disease & GP1 & GP2 & - \\
China T2D discovery & GP1 & GP2 & - \\
China T2D validation & GP1 & GP2 & - \\
China healthy Mongolian & GP1 & GP2 & GP2 \\
China ankylosing spondylitis & GP1 & & - \\
\hline
\end{tabular}

This is a provisional file, not the final typeset article 
294 Groups in the same column were considered to belong to one enterotype.

295 [insert figure 5 here]

296 Enterotypes are useful for describing the gut microbial community, and determining the association between diseases and enterotype is important to detect high risk individual in population. In the liver cirrhosis project, individuals could be broadly divided into three categories (Figure 5A). Enterotypes 1 and 3 were enriched in healthy individuals and patients, respectively ( 69 controls/16 cases vs. 2 controls/64 cases, Supplementary Table 6), and enterotype 2 accounted for half of them (43 controls, 43 cases, Supplementary Table 6). We found that the viral enterotype was significantly related to liver cirrhosis (Fisher's exact test, $\mathrm{p}=5.99 \mathrm{E}-24$, Supplementary Table 7). Enterotype 3 was loosely distributed in individuals (Figure 5A). However, enterotypes 1 and 2 showed a closer relationship. These three groups did not have discrete clustering boundaries and demonstrated some overlap with one another in the PCoA plot. There was no apparent clustering of samples enriched locally due to the viral count or the Shannon index (Figure 5B). In the hypertension project, the clustering boundaries of enterotypes 1 and 3 were more pronounced than those for enterotype 2 (Figure 5C), and there was no overlapping area between the two clusters. This finding was surprising because individuals in enterotype 2 had a smaller viral count and a lower Shannon index (Figure 5D). Some of them were close to enterotype 1 , while others had clusters of enterotype 3 . However, the specific vOTUs and enrichment direction of individuals in enterotype 2 showed a high consistency (Figure 4B), indicating that enterotype 2 was real. We found no significant association between the viral enterotype and hypertension (Fisher's exact test, $\mathrm{p}=0.3$, Supplementary Table 7). Gut virome community disorders showed significant differences in the cirrhosis and hypertension projects, which indicated that not all diseases caused evident ecological perturbation in the human gut. Thus, applying viral enterotypes as biomarkers for predicting clinical disease requires specific consideration.

\section{Discussion}

Recent investigations have shown that enterotypes of the human gut can be divided into two categories based on their predominant flora (Bacteroidetes/Prevotella). Their functions correspond to the digestion of meat and vegetarian food (Arumugam et al., 2011; Costea et al., 2017). However, some researchers consider that the distribution of enterotypes is not discrete, but rather gradient. This viewpoint suggests that those two enterotypes are the two endpoints of the gradient distribution of Bacteroidetes/Prevotella(Jeffery et al., 2012). This study used the DMM method to assign viral enterotypes and showed that there were two enterotypes in most projects. Viral enterotypes did not have an apparent dominant virus. An explanation for this finding may be that most human enteroviruses are dsDNA viruses. As previously reported, dsDNA viruses are less harmful than RNA virus to the human body(Dutilh et al., 2014; Camarillo-Guerrero et al., 2021). These viruses do not undergo strong selection when colonizing the human gut, and there is no dominant viral strain that can occupy the whole human intestine. Recent studies have shown two common and harmless dsDNA virus branches in the human intestine, namely crAssphage(Dutilh et al., 2014) and Gubaphage(Camarillo-Guerrero et al., 2021). These two dominant virus branches may correlate with the two main viral enterotypes observed in this study. We analyzed the abundance of vOTUs corresponding to each enterotype and found that in different projects, the OTUs and enrichment direction of a specific virus in the same viral enterotype were consistent. Therefore, existing evidence and our findings support the view of two discrete viral enterotypes. 
There was a third enterotype in several projects, but because of limited evidence, we could not conclude that it is ubiquitous in the human gut. In the hypertension project, researchers found that all specific vOTUs in the enterotype were shared with enterotypes 1 and 2. PCoA analysis also showed that individuals were located in the interconnection area, which is likely to explain a gradient distribution. In the liver cirrhosis project, 64 of the 66 samples were from patients, and the third enterotype was significantly related to patients. Unlike the hypertension project, the third enterotype in patients with liver cirrhosis was not related to the number of viruses. In other projects, viral enterotypes 1 and 2 had more specific viruses and a higher consistent enrichment direction in contrast to the rare specific viruses of viral enterotype 3 and an inconsistent enrichment direction. This result suggests that a third viral enterotype in various projects may not have belonged to the same cluster. Therefore, we cannot conclude that there was a stable presence of viral enterotype 3 in the population. We speculate that interaction between emergence of a disease and disorder of the gut virome may contribute to emergence of viral enterotype 3. Our results are in agreement with existing 350 studies on the disruption of the human gut community that accompanies disease(Wang and Jia, 2016; Yu et al., 2017; Nakatsu et al., 2018).

In this study, enterotype 1 had a higher viral count and Shannon index compared with enterotype 2. In addition to having a smaller sample distance, we speculate that enterotype 1 might have more stable viral ecological communities than enterotype 2. We found enterotype 2 had 1.38 times more patients than the one in enterotype 1 . This result suggests that a stable microbial community has a higher ability to resist the influence of external stimulations. In the cirrhosis project, enterotype 3 was characterized as being enriched in patients and having an extremely disordered gut virome. Enterotype 3 had the largest sample distance and highest number of virus species. A possible reason for this finding is that bile acid secretion in patients with liver cirrhosis is obstructed, which leads to drastic changes in the gut microbiome of the patients. This may have resulted in large-scale replacement of the virome and reduced similarity of virus species in this patient population. It is also possible that the microenvironment of viral evolution in the human body is disturbed owing to disease progression or the similarity of virus species is decreased due to a shift in the distribution of the ecological gradient. We also found a large distance in samples from the Sweden mother-child pair project, with the viral count being significantly lower than the global average level. A possible explanation for this finding is that the gut microbiome of young children is developing and has yet to reach a stable state(Derrien et al., 2019).

We determined enterotypes at the bacterial and viral levels in the China diabetes(Wang et al., 2012), and found a strong correlation between enterotypes at these two levels (China T2D discovery: $\mathrm{p}=$ 1.70E-07; China T2D validation: $p=1.58 \mathrm{E}-11$, Fisher's exact test, Supplementary Table 8). We found that enterotypes (bacterial and viral levels) were not randomly distributed and that the bacterial community had a strong selection effect on the viral community. However, bacterial- and viral-level enterotypes were not correlated or were weakly correlated with sex, age, BMI, and disease (Supplementary Table 9). This finding may be explained by the use of high-abundance bacterial and viral species for determining enterotypes. A high abundance of bacteria or viruses in the intestine is significantly related to disease. Such a high abundance directly and severely affects the human body, That is a microbial infection, not a harmonious symbiosis, which is in contrast to the current understanding of the gut community and health. Wang et al. used enterotype as a covariate in their study and proposed a useful method to stratify human gut microbiomes in MWAS, which effectively improved the power of hypothesis testing(Wang et al., 2012). Although we found a strong correlation between bacterial and viral enterotypes, they were not proven to be equivalent. Therefore, we suggest using bacterial and viral enterotypes as independent covariates in MWAS. More in-depth 
383

384

385

386

387

388

389

390

391

392

393

394

395

396

397

398

399

400

401

402

403

404

405

406

407

408

409

410

411

\section{Funding}

413 This work was financially supported by the Science Technology and Innovation Committee of

414 Shenzhen Municipality, China (SGDX20190919142801722).

\section{Conflict of Interest Statement}

416 The authors declare that the research was conducted in the absence of any commercial or financial 417 relationships that could be construed as a potential conflict of interest.

\section{Acknowledgments}

419 The authors thank many interns and former colleagues for collecting data, and their colleague Yufen 420 Huang for discussing the analysis strategy.

\section{References}


422

423

424

425

426

427

428

429

430

431

432

433

434

435

436

437

438

439

440

441

442

443

444

445

446

447

448

449

450

451

452

453

454

455

456

457

Ajami, N. J., Wong, M. C., Ross, M. C., Lloyd, R. E., and Petrosino, J. F. (2018). Maximal viral information recovery from sequence data using VirMAP. Nature Communications 9. doi:10.1038/s41467-018-05658-8.

Arumugam, M., Raes, J., Pelletier, E., Paslier, D. le, Yamada, T., Mende, D. R., et al. (2011). Enterotypes of the human gut microbiome. Nature 473, 174-180. doi:10.1038/nature09944.

Bateman, A., Martin, M. J., Orchard, S., Magrane, M., Agivetova, R., Ahmad, S., et al. (2021). UniProt: The universal protein knowledgebase in 2021. Nucleic Acids Research 49, D480D489. doi:10.1093/nar/gkaa1100.

Bolger, A. M., Lohse, M., and Usadel, B. (2014). Trimmomatic: A flexible trimmer for Illumina sequence data. Bioinformatics 30, 2114-2120. doi:10.1093/bioinformatics/btu170.

Boratyn, G. M., Schäffer, A. A., Agarwala, R., Altschul, S. F., Lipman, D. J., and Madden, T. L. (2012). Domain enhanced lookup time accelerated BLAST. Biology Direct 7. doi:10.1186/17456150-7-12.

Bray, N. L., Pimentel, H., Melsted, P., and Pachter, L. (2016). Near-optimal probabilistic RNA-seq quantification. Nature Biotechnology 34, 525-527. doi:10.1038/nbt.3519.

Camarillo-Guerrero, L. F., Almeida, A., Rangel-Pineros, G., Finn, R. D., and Lawley, T. D. (2021). Massive expansion of human gut bacteriophage diversity. Cell 184, 1098-1109.e9. doi:10.1016/j.cell.2021.01.029.

Costea, P. I., Hildebrand, F., Manimozhiyan, A., Bäckhed, F., Blaser, M. J., Bushman, F. D., et al. (2017). Enterotypes in the landscape of gut microbial community composition. Nature Microbiology 3, 8-16. doi:10.1038/s41564-017-0072-8.

Daszak, P., Cunningham,', A. A., and Hyatt4, A. D. Emerging Infectious Diseases of WildlifeThreats t o Biodiversity and Human Health. Available at: www.sciencemag.org.

Delcher, A. L., Bratke, K. A., Powers, E. C., and Salzberg, S. L. (2007). Identifying bacterial genes and endosymbiont DNA with Glimmer. Bioinformatics 23, 673-679. doi:10.1093/bioinformatics/btm009.

Derrien, M., Alvarez, A. S., and de Vos, W. M. (2019). The Gut Microbiota in the First Decade of Life. Trends in Microbiology 27, 997-1010. doi:10.1016/j.tim.2019.08.001.

Ding, T., and Schloss, P. D. (2014). Dynamics and associations of microbial community types across the human body. Nature 509, 357-360. doi:10.1038/nature13178.

Dutilh, B. E., Cassman, N., McNair, K., Sanchez, S. E., Silva, G. G. Z., Boling, L., et al. (2014). A highly abundant bacteriophage discovered in the unknown sequences of human faecal metagenomes. Nature Communications 5. doi:10.1038/ncomms5498.

Eric Wommack, K., Bhavsar, J., Polson, S. W., Chen, J., Dumas, M., Srinivasiah, S., et al. (2012). VIROME: A standard operating procedure for analysis of viral metagenome sequences. Standards in Genomic Sciences 6, 427-439. doi:10.4056/sigs.2945050.

This is a provisional file, not the final typeset article 
Fujimoto, K., Kimura, Y., Shimohigoshi, M., Satoh, T., Sato, S., Tremmel, G., et al. (2020). Metagenome Data on Intestinal Phage-Bacteria Associations Aids the Development of Phage Therapy against Pathobionts. Cell Host and Microbe 28, 380-389.e9. doi:10.1016/j.chom.2020.06.005.

Gregory, A. C., Zablocki, O., Zayed, A. A., Howell, A., Bolduc, B., and Sullivan, M. B. (2020). The Gut Virome Database Reveals Age-Dependent Patterns of Virome Diversity in the Human Gut. Cell Host and Microbe 28, 724-740.e8. doi:10.1016/j.chom.2020.08.003.

Hannigan, G. D., Meisel, J. S., Tyldsley, A. S., Zheng, Q., Hodkinson, B. P., Sanmiguel, A. J., et al. (2015). The human skin double-stranded DNA virome: Topographical and temporal diversity, genetic enrichment, and dynamic associations with the host microbiome. mBio 6. doi:10.1128/mBio.01578-15.

Holmes, I., Harris, K., and Quince, C. (2012). Dirichlet multinomial mixtures: Generative models for microbial metagenomics. PLoS ONE 7. doi:10.1371/journal.pone.0030126.

Jeffery, I. B., Claesson, M. J., O’Toole, P. W., and Shanahan, F. (2012). Categorization of the gut microbiota: Enterotypes or gradients? Nature Reviews Microbiology 10, 591-592. doi:10.1038/nrmicro2859.

Keesing, F., Belden, L. K., Daszak, P., Dobson, A., Harvell, C. D., Holt, R. D., et al. (2010). Impacts of biodiversity on the emergence and transmission of infectious diseases. Nature 468, 647-652. doi:10.1038/nature09575.

Knights, D., Ward, T. L., McKinlay, C. E., Miller, H., Gonzalez, A., McDonald, D., et al. (2014). Rethinking enterotypes. Cell Host and Microbe 16, 433-437. doi:10.1016/j.chom.2014.09.013.

Koleff, P., Gaston, K. J., and Lennon, J. J. (2003). Measuring beta diversity for presence-absence data.

Laffy, P. W., Wood-Charlson, E. M., Turaev, D., Weynberg, K. D., Botté, E. S., van Oppen, M. J. H., et al. (2016). HoloVir: A workflow for investigating the diversity and function of viruses in invertebrate holobionts. Frontiers in Microbiology 7. doi:10.3389/fmicb.2016.00822.

le Chatelier, E., Nielsen, T., Qin, J., Prifti, E., Hildebrand, F., Falony, G., et al. (2013). Richness of human gut microbiome correlates with metabolic markers. Nature 500, 541-546. doi:10.1038/nature12506.

Li, Y., Wang, H., Nie, K., Zhang, C., Zhang, Y., Wang, J., et al. (2016). VIP: An integrated pipeline for metagenomics of virus identification and discovery. Scientific Reports 6. doi:10.1038/srep23774.

Minot, S., Bryson, A., Chehoud, C., Wu, G. D., Lewis, J. D., and Bushman, F. D. (2013). Rapid evolution of the human gut virome. Proceedings of the National Academy of Sciences of the United States of America 110, 12450-12455. doi:10.1073/pnas.1300833110.

Minot, S., Sinha, R., Chen, J., Li, H., Keilbaugh, S. A., Wu, G. D., et al. (2011). The human gut virome: Inter-individual variation and dynamic response to diet. Genome Research 21, 16161625. doi:10.1101/gr.122705.111. 
Nakatsu, G., Zhou, H., Wu, W. K. K., Wong, S. H., Coker, O. O., Dai, Z., et al. (2018). Alterations in Enteric Virome Are Associated With Colorectal Cancer and Survival Outcomes. Gastroenterology 155, 529-541.e5. doi:10.1053/j.gastro.2018.04.018.

Paez-Espino, D., Chen, I. M. A., Palaniappan, K., Ratner, A., Chu, K., Szeto, E., et al. (2017). IMG/VR: A database of cultured and uncultured DNA viruses and retroviruses. Nucleic Acids Research 45, D457-D465. doi:10.1093/nar/gkw1030.

Paez-Espino, D., Eloe-Fadrosh, E. A., Pavlopoulos, G. A., Thomas, A. D., Huntemann, M., Mikhailova, N., et al. (2016). Uncovering Earth's virome. Nature 536, 425-430. doi:10.1038/nature19094.

Paez-Espino, D., Roux, S., Chen, I. M. A., Palaniappan, K., Ratner, A., Chu, K., et al. (2019). IMG/VR v.2.0: An integrated data management and analysis system for cultivated and environmental viral genomes. Nucleic Acids Research 47, D678-D686. doi:10.1093/nar/gky1127.

Qin, J., Li, R., Raes, J., Arumugam, M., Burgdorf, K. S., Manichanh, C., et al. (2010). A human gut microbial gene catalogue established by metagenomic sequencing. Nature 464, 59-65. doi:10.1038/nature08821.

Rampelli, S., Soverini, M., Turroni, S., Quercia, S., Biagi, E., Brigidi, P., et al. (2016). ViromeScan: A new tool for metagenomic viral community profiling. BMC Genomics 17. doi:10.1186/s12864-016-2446-3.

Reddy, T. B. K., Thomas, A. D., Stamatis, D., Bertsch, J., Isbandi, M., Jansson, J., et al. (2015). The Genomes OnLine Database (GOLD) v.5: A metadata management system based on a four level (meta)genome project classification. Nucleic Acids Research 43, D1099-D1106. doi:10.1093/nar/gku950.

Ren, J., Ahlgren, N. A., Lu, Y. Y., Fuhrman, J. A., and Sun, F. (2017). VirFinder: a novel k-mer based tool for identifying viral sequences from assembled metagenomic data. Microbiome 5, 69. doi:10.1186/s40168-017-0283-5.

Roux, S., Enault, F., Hurwitz, B. L., and Sullivan, M. B. (2015). VirSorter: Mining viral signal from microbial genomic data. PeerJ 2015. doi:10.7717/peerj.985.

Roux, S., Páez-Espino, D., Chen, I. M. A., Palaniappan, K., Ratner, A., Chu, K., et al. (2021). IMG/VR v3: An integrated ecological and evolutionary framework for interrogating genomes of uncultivated viruses. Nucleic Acids Research 49, D764-D775. doi:10.1093/nar/gkaa946.

Segata, N., Waldron, L., Ballarini, A., Narasimhan, V., Jousson, O., and Huttenhower, C. (2012). Metagenomic microbial community profiling using unique clade-specific marker genes. Nature Methods 9, 811-814. doi:10.1038/nmeth.2066.

Soneson, C., Love, M. I., and Robinson, M. D. (2015). Differential analyses for RNA-seq: transcriptlevel estimates improve gene-level inferences. F1000Research 4, 1521. doi:10.12688/f1000research.7563.1. 
533

534

535

536

537

538

539

540

541

542

543

544

545

546

547

548

549

550
Tithi, S. S., Aylward, F. O., Jensen, R. v., and Zhang, L. (2018). FastViromeExplorer: A pipeline for virus and phage identification and abundance profiling in metagenomics data. PeerJ 2018. doi:10.7717/peerj.4227.

Truong, D. T., Franzosa, E. A., Tickle, T. L., Scholz, M., Weingart, G., Pasolli, E., et al. (2015). MetaPhlAn2 for enhanced metagenomic taxonomic profiling. Nature Methods 12, 902-903. doi:10.1038/nmeth.3589.

Wang, J., and Jia, H. (2016). Metagenome-wide association studies: Fine-mining the microbiome. Nature Reviews Microbiology 14, 508-522. doi:10.1038/nrmicro.2016.83.

Wang, J., Qin, J., Li, Y., Cai, Z., Li, S., Zhu, J., et al. (2012). A metagenome-wide association study of gut microbiota in type 2 diabetes. Nature 490, 55-60. doi:10.1038/nature11450.

Yu, T. C., Guo, F., Yu, Y., Sun, T., Ma, D., Han, J., et al. (2017). Fusobacterium nucleatum Promotes Chemoresistance to Colorectal Cancer by Modulating Autophagy. Cell 170, 548563.e16. doi:10.1016/j.cell.2017.07.008.

Zuo, T., Sun, Y., Wan, Y., Yeoh, Y. K., Zhang, F., Cheung, C. P., et al. (2020). Human-Gut-DNA Virome Variations across Geography, Ethnicity, and Urbanization. Cell Host and Microbe 28, 741-751.e4. doi:10.1016/j.chom.2020.08.005. 
A

.

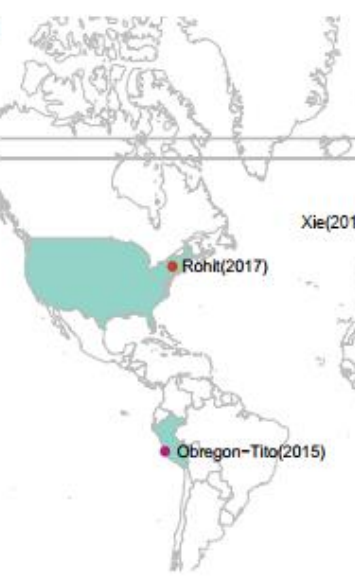

.
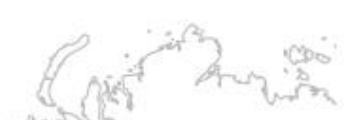

- Karisson(2013)

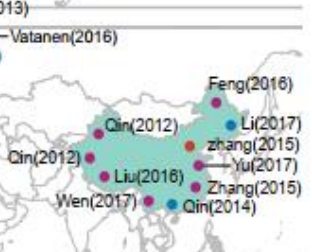

B

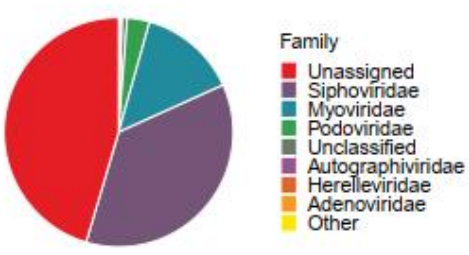

Reported enterotypes

- One type

- Twroe types

$\mathrm{C}$

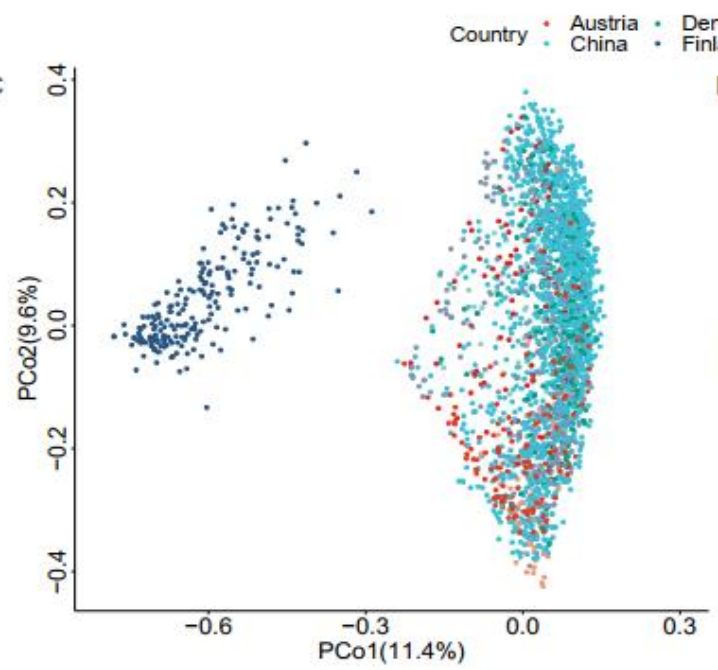

Country : Austria : Denmark : Peru China $:$ UK

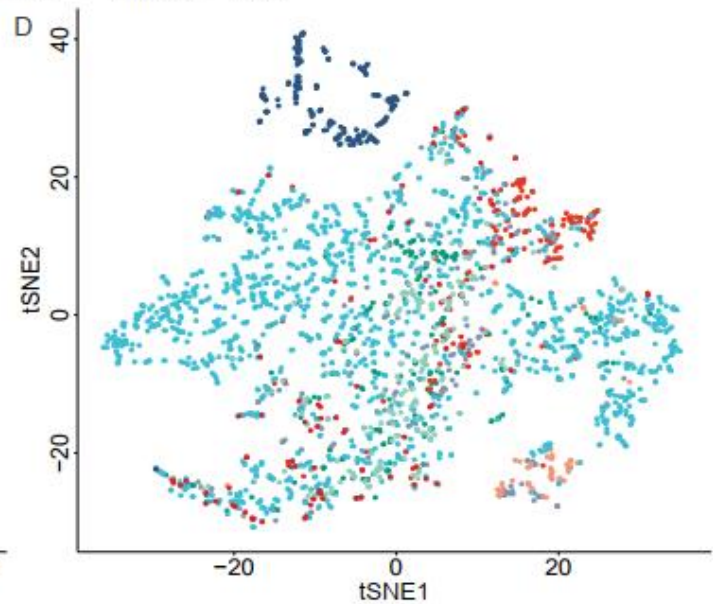

552 Figure 1: Location, taxonomic assignment, and abundance of the 2,690 samples. A: Geographic locations of the 18 projects, with classification by the number of enterotypes. B: Pie chart shows viral taxonomic assignment at the family level by protein alignment. C: Principal Coordinates Analysis (PCoA) plot based on the Bray-Curtis distance and the relative abundance of viruses. D: tDistributed Stochastic Neighbor Embedding (tSNE) plot based on the relative abundance of viruses. 
bioRxiv preprint doi: https://doi.org/10.1101/2021.07.26.453761; this version posted July 26, 2021. The copyright holder for this preprint (which was not certified by peer review) is the author/funder, who has granted bioRxiv a license to display the preprint in perpetuity. It is made

A

A 8

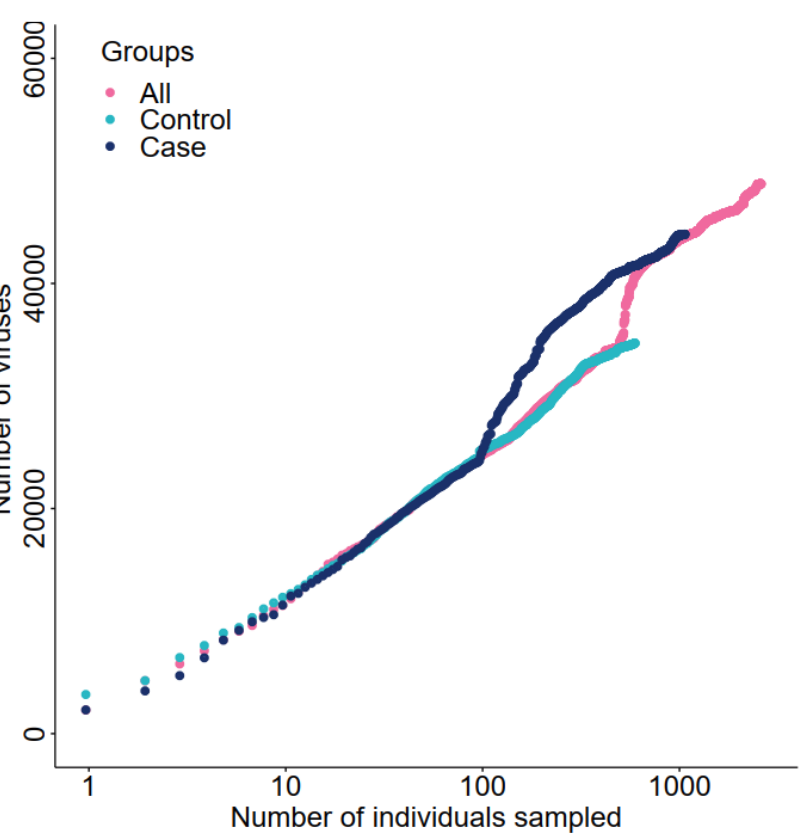

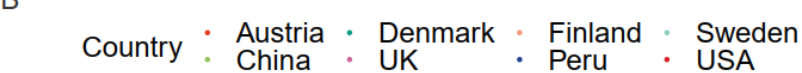

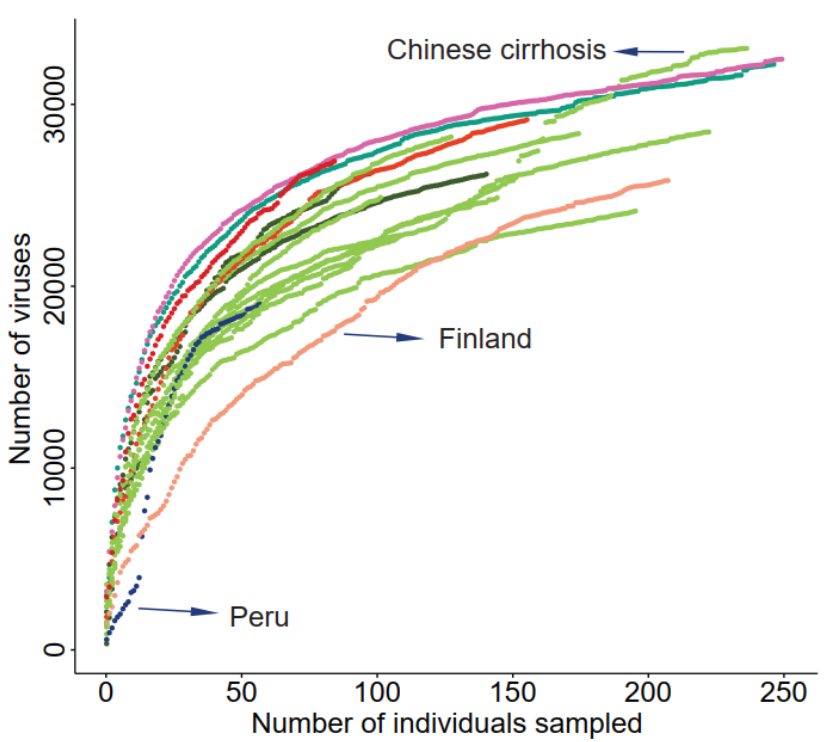

559 Figure 2: Cumulative curves of the number of virus species against the number of samples. A:

560 Cumulative curves of cases, controls, and all samples. Only samples from studies with a case-control 561 design were included. B: Cumulative curves of sample data divided into seven countries. 
A

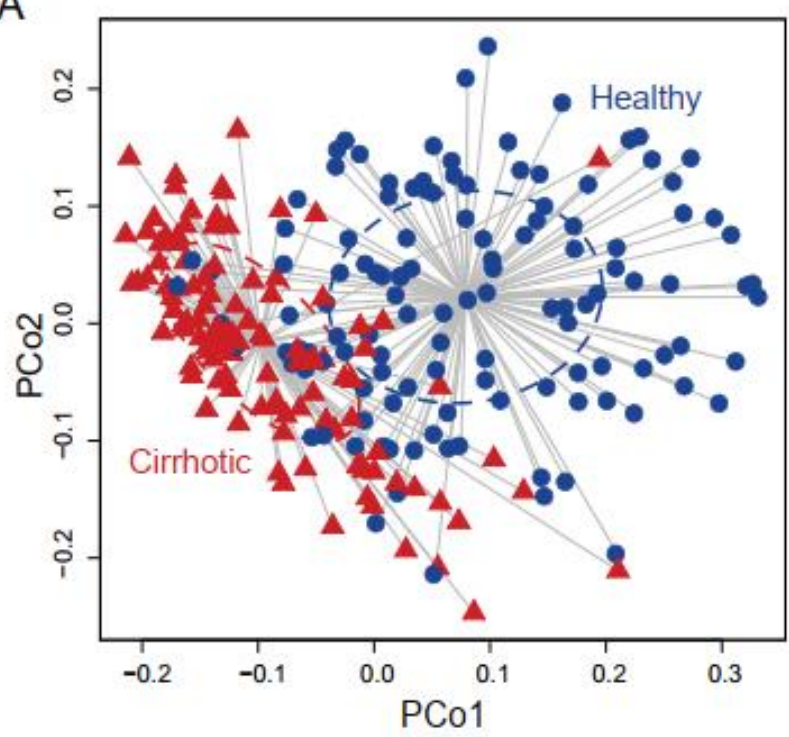

C

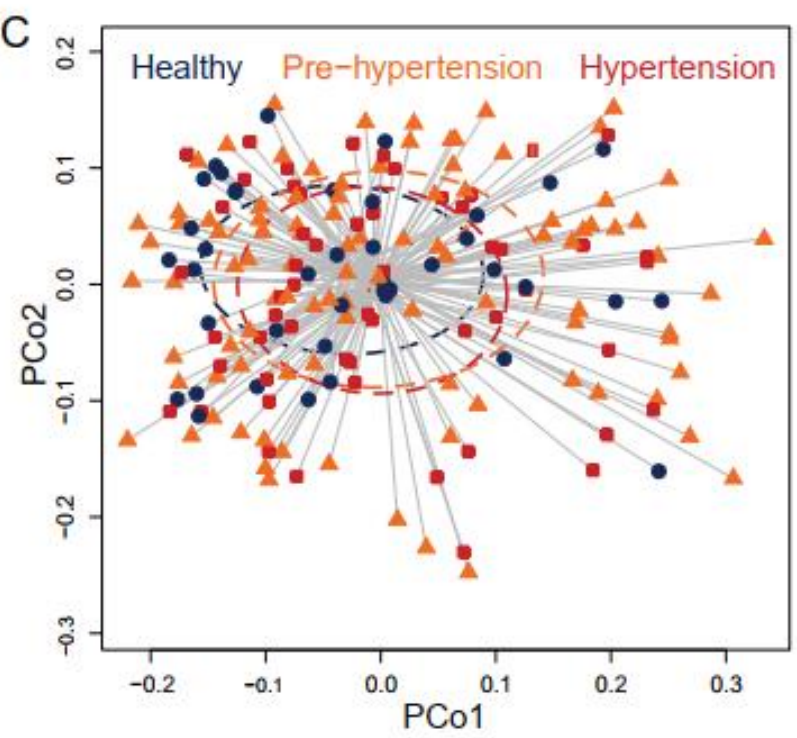

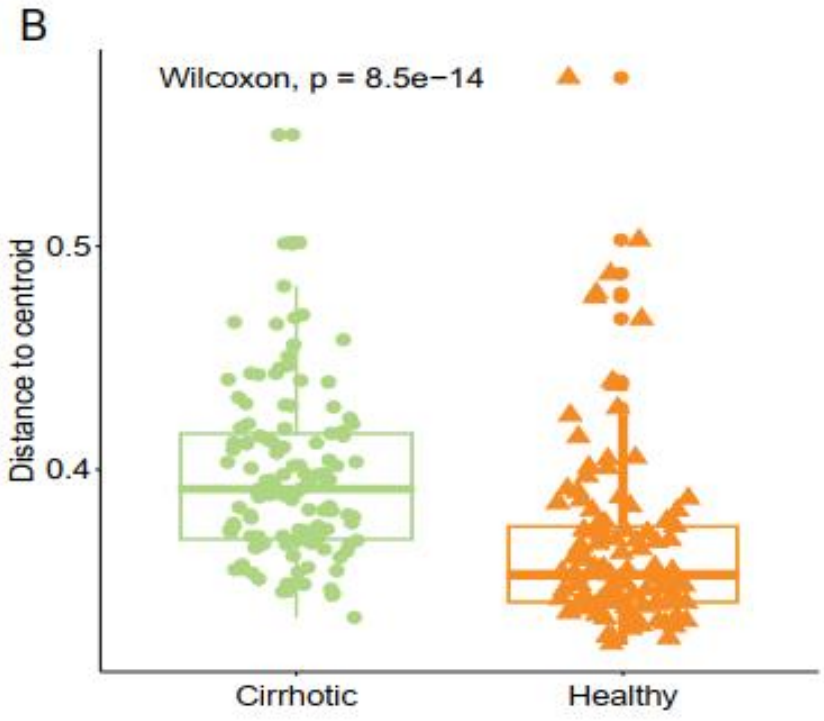

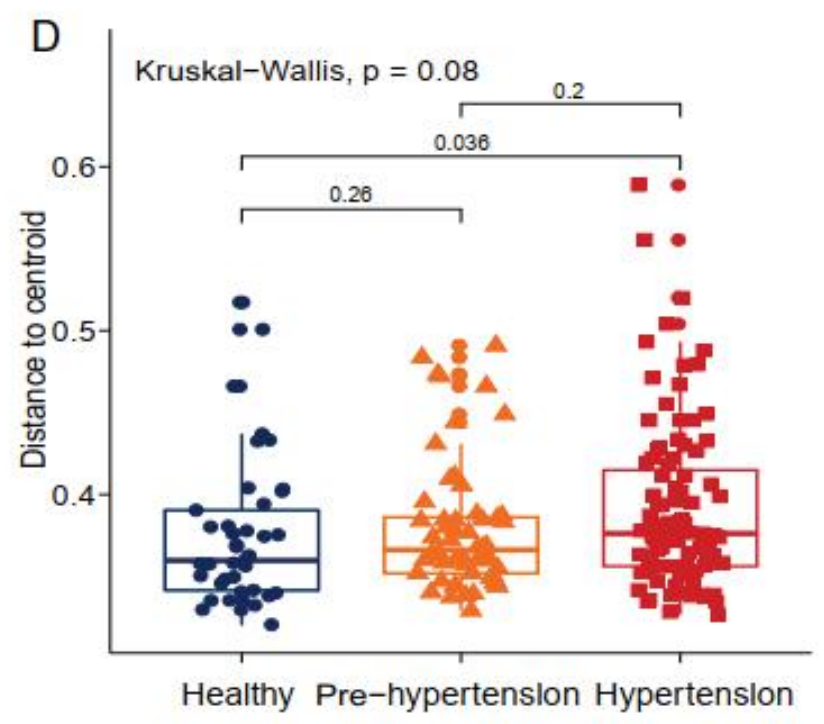

564 Figure 3: Gut virome characterized by beta diversity in the included projects. (A) Principal coordinates analysis plot of the cirrhosis project. Each ellipse represents a cohort, and the point connected by the straight gray lines represents the centroid. (B) Boxplot of the distance to the centroid. A significant difference in the distance to the centroid was found between the two groups. (C) Principal coordinates analysis plot of the hypertension project. (D) Boxplot of the hypertension project with comparison for each pair of the three groups. 

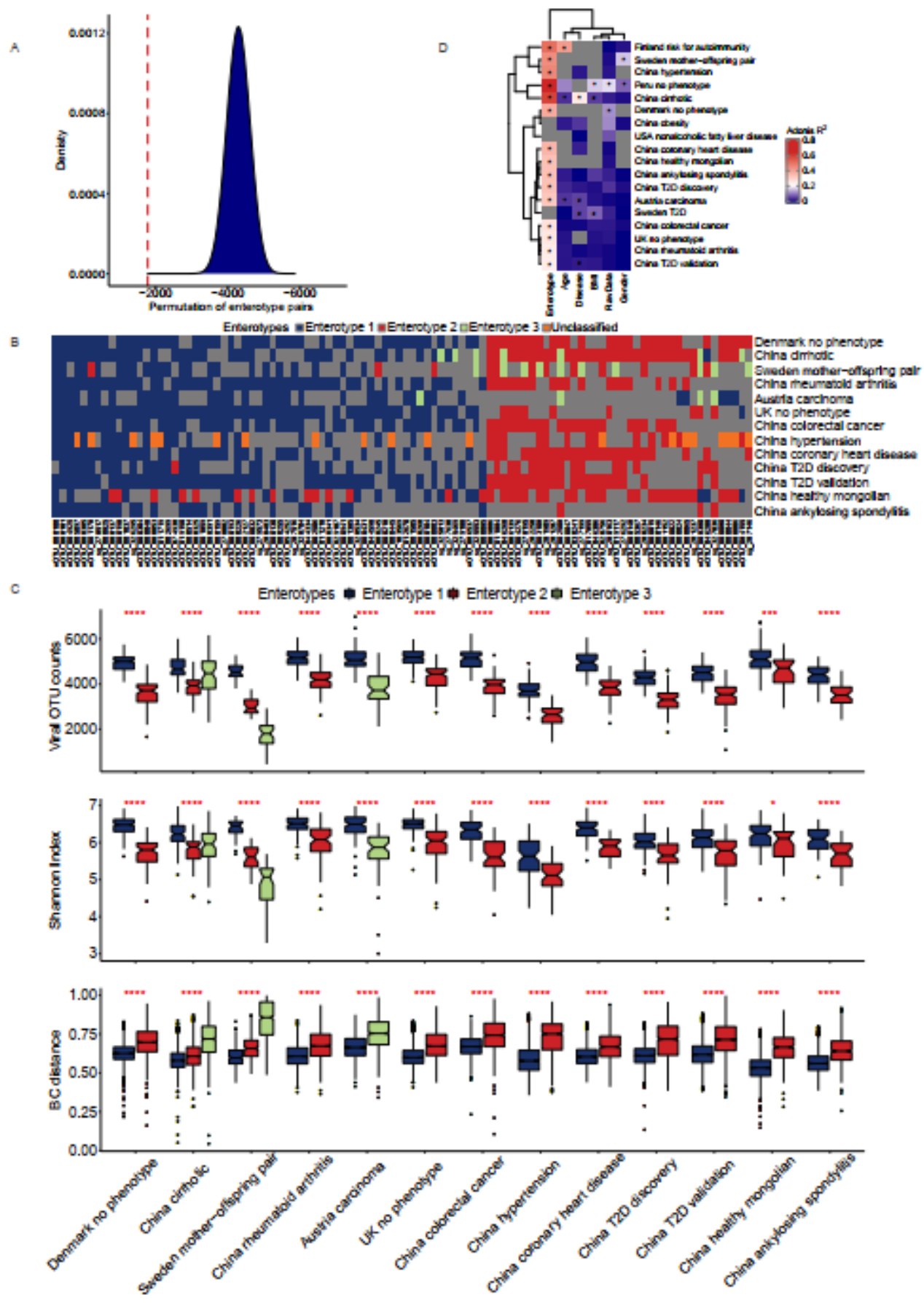

572 Figure 4: Characterization of viral enterotypes in all projects. A: We used the random pairing method to confirm the accuracy of artificial enterotype classification. The density map shows the score distribution of 5 million permutations, and the red line indicates the score of the manual category. B: The categories of manual enterotypes in different projects show a high concordance of their specific vOTUs and enrichment direction. C: Ecological diversity of different viral enterotype populations. D: Effect of different covariates on the structural variance of the gut virome community. 
A

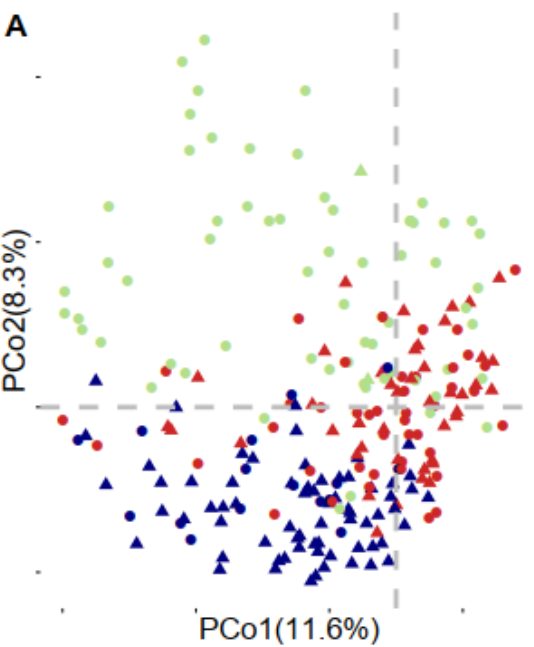

C

C

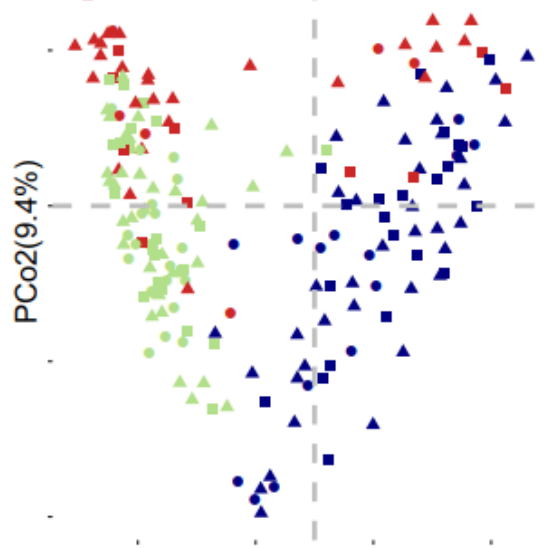

$\operatorname{PC} 1(19.9 \%)$
Phenotype

- Cirrhotic

- Healthy

Enterotypes

- Enterotype 1

- Enterotype 2

- Enterotype 3

Phenotype

- Healthy

- Hypertension

- Pre-hypertension

Enterotypes

- Enterotype 1

- Enterotype 2

- Enterotype 3
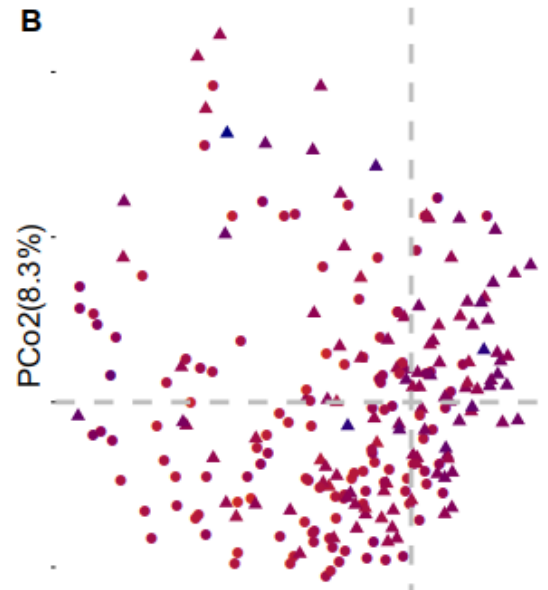

'PCo1(11.6\%)

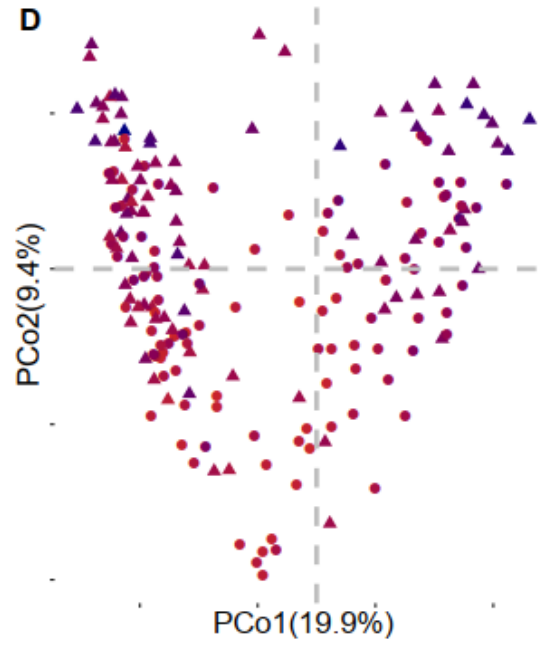

Shannon

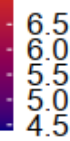

Count

- High

- Low
- High

- Low

Shannon

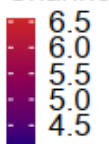

580 Figure 5: Detailed PCoA map of liver cirrhosis and hypertension. A: Samples of liver cirrhosis were plotted in relation to their phenotype and enterotypes. B: Samples of liver cirrhosis were plotted in relation to their viral count and Shannon index. C: Samples of hypertension were plotted in relation to their phenotype and enterotype. D: Samples of hypertension were plotted in relation to their viral count and Shannon index. 